\title{
Sanjaya Lall: la competitividad industrial y las capacidades tecnológicas
}

\author{
Daniel Osorio Barreto * \\ Carlos David Cardona Arenas ** \\ Cristhian Guillermo Naranjo Herrera ${ }^{* * *}$
}

Fecha de recepción: 21 de febrero de 2020

Fecha de aprobación: 15 de septiembre de 2020

Resumen: Este artículo busca identificar el desarrollo teórico y empírico del campo de investigación de la competitividad industrial en torno al concepto de capacidades tecnológicas propuesto por Sanjaya Lall en el marco de las firmas. Se emplea una metodología que consiste en la revisión sistemática de literatura durante el periodo comprendido entre 1987 y 2019. Como principal hallazgo, el estudio permitió identificar claramente tres líneas futuras de investigación sobre la dinámica de las capacidades tecnológicas, la estructura de incentivos y el análisis de las variables macrofundamentales, lo que permite concluir que existe un campo de estudio emergente con relación a las interacciones entre las capacidades tecnológicas, desde su perspectiva económica, y los recursos y capacidades desde la literatura estratégica y administrativa. Con lo cual este estudio aporta de manera significativa a la reflexión teórica en la relación que existe entre las capacidades tecnológicas y la gestión, las métricas de los recursos de la firma y los escenarios, ambientes y procesos de aprendizaje en el ámbito individual, colectivo, organizativo e interorganizativo de las firmas.

Palabras clave: capacidades tecnológicas; industrialización; innovación; crecimiento económico; ventajas competitivas; firma.

Clasificación JEL: L0, L2, D4, M2.

Cómo citar este artículo:

Osorio-Barreto, D., Cardona-Arenas, C., \& Naranjo Herrera, C. (2021). Sanjaya Lall: la competitividad industrial y las capacidades tecnológicas. Apuntes del Cenes, 40(71). Págs. 41 - 74.

https://doi.org/10.19053/01203053.v40.n71.2021.10659

* M.C. en Economía . Profesor asistente del Departamento de Administración y Economía de la Universidad Autónoma de Manizales, Colombia. Adscrito al Grupo de Investigación en Empresariado. dosoriob@autonoma.edu.co (iDhttps://orcid.org/0000-0003-0848-6394

** M.C. en Economía. Profesor asistente del Departamento de Administración y Economía de la Universidad Autónoma de Manizales, Colombia. Adscrito al Grupo de Investigación en Empresariado. ccardonaa@autonoma.edu.co (iD https://orcid.org/0000-0003-0089-1109 (Autor de correspondencia)

*** Ph. D. en Administración de Empresas y Dirección de Recursos Humanos. Profesor asociado del Departamento de Administración y Economía de la Universidad Autónoma de Manizales, Colombia. Adscrito al Grupo de Investigación en Empresariado. cnaranjo@autonoma.edu.co iD https://orcid.org/0000-0002-2886-0067 


\title{
Sanjaya Lall: Industrial Competitiveness and Technological Capabilities
}

\begin{abstract}
This paper seeks to identify the theoretical and empirical development of the research field of industrial competitiveness on the concept of technological capabilities proposed by Sanjaya Lall in the framework of firms. A methodology consisting of the systematic review of literature during the period between 1987 and 2019 is proposed. As the main finding, the study clearly identified three future research lines on technological capabilities dynamics, structure incentives and the analysis of macro-fundamental variables, which allows to conclude that there is an emerging field of study in relation to the interactions between technological capabilities, from its economic perspective, and resources and capabilities from the strategic and administrative literature. So, this study contributes significantly to the theoretical reflection on the relationship between technological capabilities and management, the metrics of the firm's resources, and the scenarios, environments and learning processes at firm's individual, collective, organizational and interorganizational level.
\end{abstract}

Keywords: technological capabilities; industrialization; innovation; economic growth; competitive advantages; firm. 


\section{INTRODUCCIÓN}

Este trabajo tiene como objetivo identificar el desarrollo teórico y empírico del campo de investigación sobre la competitividad industrial a través de la aproximación al concepto de capacidades tecnológicas, atendiendo la necesidad del campo de investigación de la economía aplicada. Existen diversas perspectivas teóricas para comprender los determinantes del proceso de generación de valor agregado, aumento de la competitividad y resultados en el crecimiento económico. Se destacan trabajos seminales como los de Ramsey (1928), Solow (1956), Swan (1956), Koopmans (1965), Usawa (1965), Lucas (1988, 1990), Porter (1990), Barro (1991), Rebelo (1991), Barro y Lee (1994), Barro y Sala-i-Martin (2004), entre otros. Las corrientes desde la teoría económica se originan en los desarrollos teóricos propuestos por Kaldor (1970), quien propone una causación acumulativa positiva entre inversión-productividad-exportaciones-crecimiento económico, postura teórica que abre una línea de investiga- ción asociada a los determinantes del crecimiento económico de las naciones, centrada en el fortalecimiento industrial.

Para Dutrénit et al. (2019), las capacidades tecnológicas propenden a lograr el uso efectivo del conocimiento tecnológico para propiciar la innovación y la inversión efectiva a fin de optimizar la producción; posición consistente con la noción de capacidad tecnológica proporcionada por García y Navas (2007) y por Ince et al. (2016), quienes concuerdan en que las capacidades tecnológicas permiten el uso y aprovechamiento de los recursos físicos, financieros, humanos, tecnológicos, de sistemas de información y de comunicación como aporte a la generación de beneficios económicos que incentivan la reinversión para adaptarse a los retos tecnológicos por medio estrategias de innovación y así producir ventajas competitivas.

El campo de investigación con relación a la acumulación de capacidades tecnológicas recientemente se ha planteado como un debate central en la literatura de la economía aplicada a las organizaciones, debido a su importancia en la 
comprensión de los determinantes del desempeño económico de estas y del logro de la competitividad empresarial (Hansen \& Lema, 2019). Así, la competitividad es el elemento articulador, entendido como la capacidad de generar valor agregado de manera sostenida y sostenible en el tiempo. Desde esta perspectiva se tienen en cuenta los niveles de la competitividad descritos por Chica (2007a, 2007b) desde una óptica sistémica, que articula la firma, el sector/región y la nación con la acumulación de capacidades, como el principal motor del sistema de causación acumulada; esto en consonancia con la propuesta original de Lall (1992, 2001) donde las capacidades tecnológicas son el elemento clave en la firma para la competitividad. En este sentido, Lall (2001) define las capacidades tecnológicas como las habilidades de usar de forma efectiva el conocimiento tecnológicol. Con lo cual queda claro el interés aplicativo para la toma de desiciones empresariales y articulado con las derivaciones más recientes con origen en Lall (2001), en términos, por ejemplo, de cambios tecnológicos más actualizados, ligados al enfoque recursos y capacidades.
En el enfoque de capacidades tecnológicas de Lall (1991, 1992, 2001) se aborda la competitividad desde una perspectiva industrial, en coherencia con los aportes de Kaldor (1961, 1970, 1975) sobre el fortalecimiento industrial como motor esencial del crecimiento económico, donde es definida como la capacidad de los países para aumentar su presencia en mercados domésticos e internacionales mientras desarrollan sus sectores industriales y sus actividades con alto valor agregado y un alto nivel de tecnología (Lall, 1992).

En este sentido, se pretende identificar el desarrollo teórico y empírico del campo de investigación de la competitividad industrial en torno al concepto de capacidades tecnológicas propuesto por Sanjaya Lall, en el marco de las firmas, con el fin de analizar las diferentes estrategias que pueden surgir dentro de las organizaciones para elevar sus niveles de competitividad a partir de la acumulación de dichas capacidades. Por lo tanto, los objetivos específicos son: i) examinar el estado del conocimiento y las evidencias empíricas de la investigación sobre el concepto de capacidades tecnológicas a través de una estrategia de búsqueda y ii) identificar líneas futu-

1 Partiendo de la definición propuesta por Lall (2001), Gonsen (1998) amplia su descripción como "la capacidad de seleccionar, asimilar, adaptar y mejorar la tecnología existente o importada y/o creando nueva tecnología, a partir de un uso efectivo del conocimiento tecnológico" (p. 7). De esta manera, las capacidades tecnológicas se pueden desagregar en dos procesos: i) adquisición de la capacidad, asociada a la búsqueda de las tecnologías disponibles junto con sus alternativas y su apropiada selección y, ii) capacidad adaptativa, referida a la adaptación de la tecnología a las condiciones específicas de los procesos productivos. Adicionalmente, Enos (1991) destaca que desde esta perspectiva las capacidades tecnológicas tienen dos agentes: los individuos, quienes poseen las habilidades y el conocimiento técnico y la organización, comprendida como el conjunto de instituciones que reúnen los diferentes talentos potenciados por el know-how en la producción como el objetivo central hacia el cual se dirigen los esfuerzos combinados de aquellos con habilidades técnicas y conocimiento tecnológico. 
ras de investigación acerca del campo de la competitividad industrial en relación con dicho concepto.

Chica (2007a) destaca que el eje articulador de la dinámica de la competitividad es la acumulación de capacidades dentro de las firmas, de tal manera que capture elementos organizacionales, institucionales y sistémicos que pueden ser omitidos por posturas reduccionistas que asocian exclusivamente a la competitividad con la productividad, los cuales van de la mano de procesos de acumulación de capital en un sentido amplio: capital fijo, capital financiero, capital en conocimiento, capital organizacional. En la medida que se presente un proceso de acumulación de capacidades dentro de la firma (nivel micro) se potenciará la dinámica de inversión, el cambio técnico y el posicionamiento en el mercado apoyado en las dos formas genéricas de competitividad que fortalecerán la red institucional descrita en el nivel meso y que permitirán tener mayor conciencia de los aspectos fundamentales que inciden en el nivel macro. Así, Chica (2007a) afirma que el concepto central de esta visión sistémica de la competitividad es la acumulación de capacidades, y resalta que unos de los autores que más ha contribuido al análisis económico de la competitividad es Sanjaya Lall con el enfoque de capacidades tecnológicas.

De manera específica, Lall (1992) sostiene que en el ámbito de la firma, las capacidades tecnológicas pueden comprenderse desde los siguientes pilares: capacidades de inversión, capacidades de producción y capacidades de vinculación.

- Capacidades de inversión: se asocian con las "habilidades necesarias para identificar, preparar y obtener tecnología para diseñar, construir, equipar, proveer de personal y/o encargar una nueva instalación (o expansión)" (Lall, 1992, p. 168). Estas capacidades determinan el costo de capital de un proyecto, la adecuación de la escala de producción, la tecnología y equipos utilizados $y$, finalmente, el entendimiento por parte de la firma a la hora de poner en marcha la tecnología disponible, lo que incide en la eficiencia.

- Capacidades de producción: estas capacidades abarcan "desde habilidades básicas como control de calidad, operación y mantenimiento hasta otras más avanzadas como adaptación, mejora o 'stretching' de equipos, hasta las más exigentes de investigación, diseño e innovación" (Lall, 1992, p. 168), por lo que no solo determinan qué tan bien las tecnologías disponibles son operadas y mejoradas, sino también los esfuerzos internos asociados a la absorción de las tecnologías adquiridas recientemente o imitadas por otras firmas.

- Capacidades de vinculación: definidas como las "habilidades necesarias para transmitir información, habilidades y tecnología desde y para la empresa (a lo largo de la cadena de valor)" (Lall, 1992, p. 168), que no 
solo afectan la eficiencia productiva de la empresa sino también la posibilidad de generar externalidades positivas (efectos derrame) en firmas pertenecientes a la misma industria o a sectores complementarios.
De la mano de las definiciones presentadas, en la siguiente tabla se ilustra la dinámica de las capacidades tecnológicas según su nivel de complejidad:

Tabla 1. Matriz de capacidades tecnológicas

\begin{tabular}{|c|c|c|c|c|c|c|c|}
\hline & & \multicolumn{2}{|c|}{ Inversión } & \multicolumn{3}{|c|}{ Producción } & \multirow[b]{2}{*}{ Vinculación } \\
\hline & & Pre inversión & $\begin{array}{c}\text { Ejecución del } \\
\text { proyecto }\end{array}$ & $\begin{array}{l}\text { Ingeniería de } \\
\text { procesos }\end{array}$ & $\begin{array}{l}\text { Ingeniería de } \\
\text { producto }\end{array}$ & $\begin{array}{l}\text { Ingeniería } \\
\text { industrial }\end{array}$ & \\
\hline 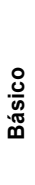 & $\begin{array}{l}\text { Simple, rutina } \\
\text { (basada en la } \\
\text { experiencia) }\end{array}$ & $\begin{array}{l}\text { Estudios de } \\
\text { prefactibilidad } \\
\text { y factibilidad } \\
\text { selección de la } \\
\text { ubicación, pro- } \\
\text { gramación de la } \\
\text { inversión }\end{array}$ & $\begin{array}{l}\text { Construcción } \\
\text { civil, servicios } \\
\text { auxiliares, } \\
\text { montaje del } \\
\text { equipo, puesta } \\
\text { en marcha }\end{array}$ & $\begin{array}{l}\text { D e p u r a c i ó n, } \\
\text { equilibrio, control } \\
\text { p r e v e n t i vo, } \\
\text { control de calidad, } \\
\text { asimilación de } \\
\text { la tecnología de } \\
\text { acceso }\end{array}$ & $\begin{array}{l}\text { Asimilación de } \\
\text { diseño de produc- } \\
\text { tos, adaptaciones } \\
\text { menores frente a } \\
\text { necesidades del } \\
\text { mercado }\end{array}$ & $\begin{array}{l}\text { Flujo de trabajo, } \\
\text { control de } \\
\text { inventarios, } \\
\text { programación, } \\
\text { estudio de tiempo } \\
\text { y movimientos }\end{array}$ & $\begin{array}{l}\text { Obtención local } \\
\text { de bienes y servi- } \\
\text { cios, intercambio } \\
\text { de información } \\
\text { con proveedores }\end{array}$ \\
\hline 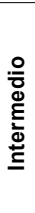 & $\begin{array}{l}\text { A d a p t a t i - } \\
\text { va-Duplicativa } \\
\text { (basada en la } \\
\text { búsqueda) }\end{array}$ & $\begin{array}{l}\text { Búsqueda de } \\
\text { fuentes tecnológi- } \\
\text { cas negociación } \\
\text { de contratos, } \\
\text { negociación de } \\
\text { términos adecua- } \\
\text { dos, información } \\
\text { de sistemas }\end{array}$ & $\begin{array}{l}\text { Adquisición de } \\
\text { equipos, inge- } \\
\text { niería detallada, } \\
\text { reclutamiento } \\
\text { de personal } \\
\text { calificado }\end{array}$ & $\begin{array}{l}\text { Equipo de exten- } \\
\text { sión, adaptación y } \\
\text { ahorro de costos, } \\
\text { licenciamiento de } \\
\text { nueva tecnología }\end{array}$ & $\begin{array}{l}\text { Mejoramiento } \\
\text { de la calidad del } \\
\text { producto, licen- } \\
\text { ciamiento y } \\
\text { asimilación de } \\
\text { nuevas tecnologías } \\
\text { importadas }\end{array}$ & $\begin{array}{l}\text { Monitoreo de la pro- } \\
\text { ductividad, mejora } \\
\text { en coordinación }\end{array}$ & $\begin{array}{l}\text { Transferencia de } \\
\text { tecnología a pro- } \\
\text { veedores locales, } \\
\text { coordinación de } \\
\text { diseños, vínculos } \\
\text { de ciencia y } \\
\text { tecnología }\end{array}$ \\
\hline 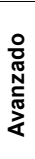 & $\begin{array}{l}\text { I n n o va do - } \\
\text { ra-arriesgada } \\
\text { (basada en la } \\
\text { investigación) }\end{array}$ & & $\begin{array}{l}\text { Procesos bási- } \\
\text { cos de diseño, } \\
\text { diseño y suminis- } \\
\text { tro de equipos }\end{array}$ & $\begin{array}{l}\text { Innovación interna } \\
\text { de procesos, } \\
\text { investigación } \\
\text { básica }\end{array}$ & $\begin{array}{l}\text { Innovación interna } \\
\text { de productos, } \\
\text { investigación } \\
\text { básica }\end{array}$ & & $\begin{array}{l}\text { Capacidades } \\
\text { de cooperación } \\
\text { en I+D, licen- } \\
\text { ciamiento de } \\
\text { propia tecnología } \\
\text { a terceros }\end{array}$ \\
\hline
\end{tabular}

Fuente: traducción propia con base en Lall (1991).

Así, los diferentes tipos de capacidades tecnológicas contribuyen a la gestión del conocimiento de la empresa de la mano de la acumulación de capital (humano, físico, organizacional, en conocimiento), para potenciar la productividad y el cambio técnico dentro de las firmas con el fin de consolidar la competitividad estratégica o de precios soportada en los determinantes meso y macro mencionados.
Cabe destacar que los pilares de las capacidades tecnológicas en el nivel de la firma son potencializados por los siguientes factores del nivel país: i) capacidades, fomento de la inversión física, el capital humano y el esfuerzo tecnológico; ii) incentivos asociados a la promoción del mercado de factores, la competencia y la estabilidad macro, $y$ iii) instituciones, reglas de juego que faciliten la acumulación de capacidades tecnológicas, industriales y fortalecimiento del talento humano. 
Con base en lo expresado es clara la conexión de esta perspectiva económica con la teoría de recursos y capacidades (Barney, 1991; Ambrosini et al., 2009; Miller, 2019) en el ámbito estratégico-administrativo, en cuanto refieren a un conjunto de recursos o activos intangibles que es necesario identificar y fortalecer mediante su adecuada gestión, como plantea el capital intelectual (Al-Jinini , 2019; De Pablos \& Edvinsson, 2020), de manera que la combinación de recursos como el talento y el conocimiento, por ejemplo, genere y dinamice diversas capacidades organizativas, como las de tipo tecnológico, en pro de alcanzar y sostener ventajas competitivas. En tal sentido, la aplicación práctica de los postulados teóricos aquí abordados, no solo es posible, sino conveniente y amplia.

\section{METODOLOGÍA}

Una vez contextualizadas las capacidades tecnológicas, basados en los aportes de Sanjaya Lall, y su relación con la competitividad industrial, se realizó la revisión sistemática de literatura desde la siguiente pregunta orientadora: ¿cuál ha sido el desarrollo teórico y metodológico sobre las capacidades tecnológicas y su relación con la industrialización, coadyuvante de la competitividad industrial, en las firmas?
A pesar de que existen trabajos anteriores sobre gestión de la tecnología de Beyhan y Cetindamar (2013), la pregunta planteada tiene como objetivo hacer una revisión de literatura que apunte a comprender la visión contemporánea del concepto de capacidades tecnológicas y su relación con la industrialización, revisando los principales artículos científicos que han continuado la reflexión teórica o empírica enfocada en análisis de casos concretos de firmas y países. A partir de lo anterior se identifican tres conectores claves para incluir en la ecuación de búsqueda (EB), la cual se aplica en las bases de datos Web of Science (WoS) y Scopus, debido a su nivel de impacto 2 y para la ventana de tiempo 1987 a 2019.

\section{Technological capabilities + Indus- trialization + Firm*}

La EB planteada pretende identificar artículos (papers) que incluyan en su título / resumen (abstract) / palabras clave (keywords) relacionados con lo propuesto. Para tal propósito, la búsqueda se realiza en las bases de datos de WoS y Scopus, en las que se obtiene la ventana de análisis 1987-2019. Específicamente, la EB arroja una clasificación muy acotada de los artículos científicos por revisar, en el sentido de que se llega a un total de 11 artículos para el caso de WoS (desde 2006 hasta 2019)

2 Cabe destacar que esta ecuación de búsqueda surge después de una depuración de conceptos sinónimos a industrialización y firmas, acorde a la estructura conceptual propuesta por Lall y los trabajos posteriores a sus aportes. Adicionalmente, la terminación en * de la tercera palabra de la ecuación de búsqueda está asociada a la posibilidad de articular otros sufijos de la misma. 
y 36 artículos para el caso de SCOPUS (desde 1987 hasta 2019); los cuales, una vez sistematizados y unificados en una base de datos, generan como resultado 40 documentos para analizar.

Para este propósito, se realizan dos tipos de análisis: i) cuantitativo, basado en la bibliometría para identificar el panorama general del estado de conocimiento en términos de revistas, autores, red de coocurrencias, palabras claves más relevantes y áreas de conocimientos sobresalientes, y ii) cualitativo, que describe y articula los trabajos identificados en la EB y su pertinencia para el campo de investigación mencionado en la primera sección. Es importante resaltar que el análisis cuantitativo estuvo basado en la herramienta Bibliometrix desarrollada por Aria y Cuccurullo (2017).

RESULTADOS: ESTADO DE CONOCIMIENTO Y EVIDENCIAS EMPÍRICAS SOBRE EL CONCEP-

\section{TO DE CAPACIDADES TECNO- LÓGICAS EN EL MARCO DE LAS FIRMAS}

\section{Análisis cuantitativo}

Cuando se aplica la EB propuesta, se encuentra una clasificación muy acotada de los artículos científicos por revisar, la cual es pertinente porque está asociada estrechamente con los propósitos de la revisión sistemática y permite revisar las publicaciones detalladamente en un marco de referencia delimitado para los propósitos de la investigación.

En relación con las áreas de conocimiento, la búsqueda en Scopus posibilita identificar las más relevantes: ciencias sociales (28.8\%); negocios, gestión y contabilidad (27.3\%); ingeniería (16.7\%); ciencia de la computación (9.1\%), economía, econometría y finanzas $(9.1 \%)$. Por otro lado, la Figura 1 evidencia el número de publicaciones y citaciones en WoS:

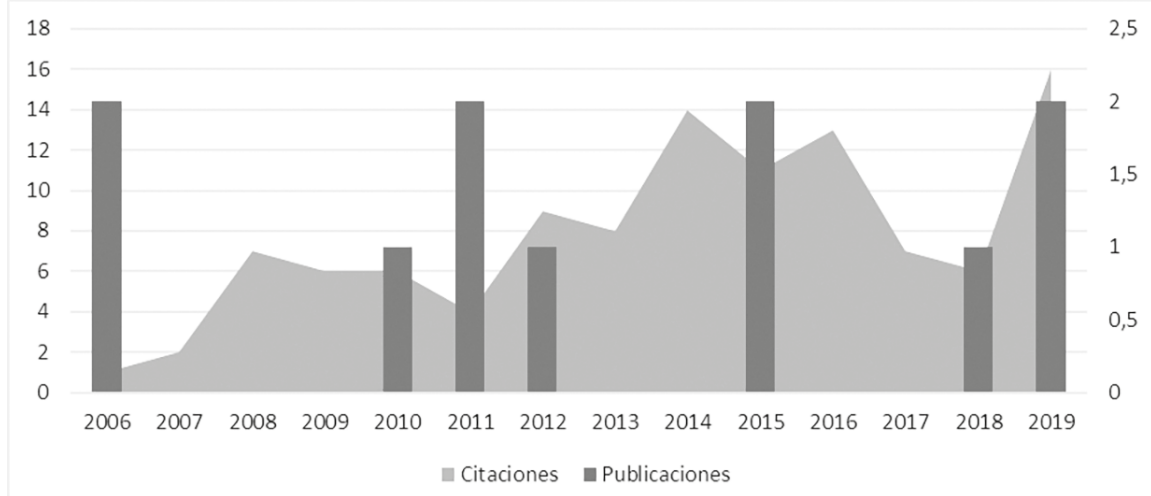

Figura1. Número de publicaciones y citaciones según la EB (WoS). 
Fuente: elaboración propia con base en WoS.

Por otro lado, en el ranking de autores tes: Lall (1992) con 1091 citaciones, según su producción científica, se Dahlman (1987) con 273 citaciones, encuentra que los autores son variados Kim (1999) con 138 citaciones, Bell y no sobresalen frente al número de artí- (2006) con 90 citaciones y Madanmohan culos publicados. En este sentido, los (2004) con 46 citaciones. La Figura 2 artículos más citados son los siguien- resume los resultados:

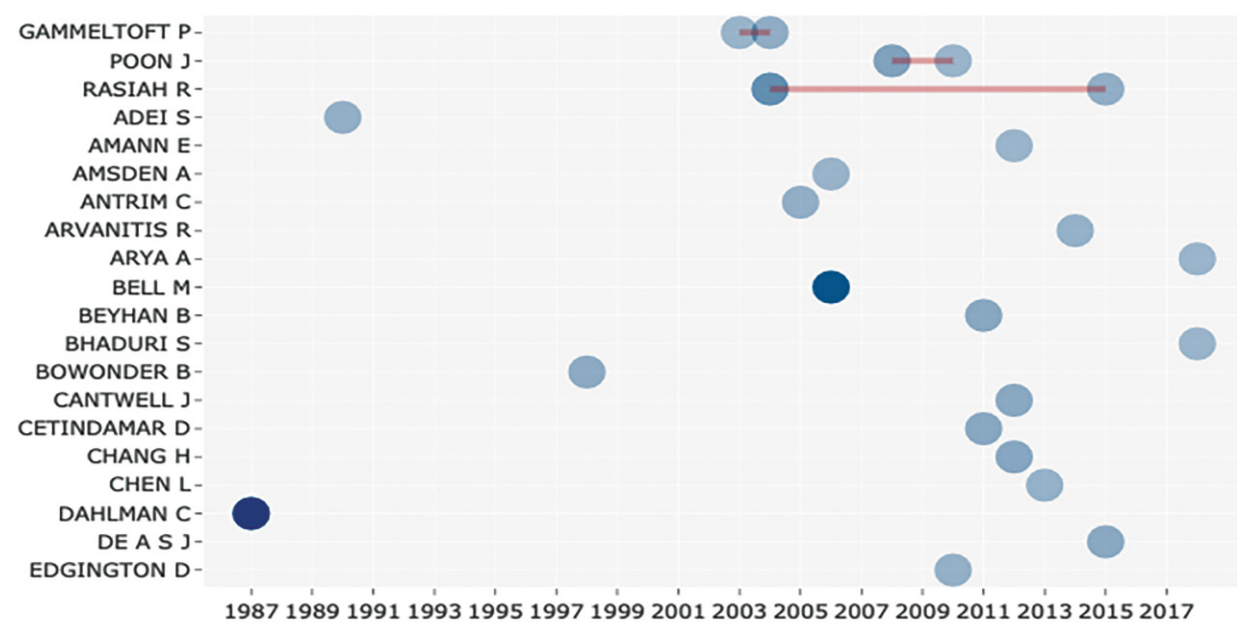

Figura 2. Ranking de autores según su producción científica

Fuente: elaboración propia con base en Aria y Cuccurullo (2017).

Nota: el tamaño del círculo es proporcional al número de artículos por año y la escala de color varía según el número de citaciones por año.

Frente a las palabras clave más desta- lógicas y la industrialización. La Figura cadas, las más frecuentes son aquellas 3 muestra el gráfico de coocurrencias relacionadas con las capacidades tecno- según las palabras clave: 


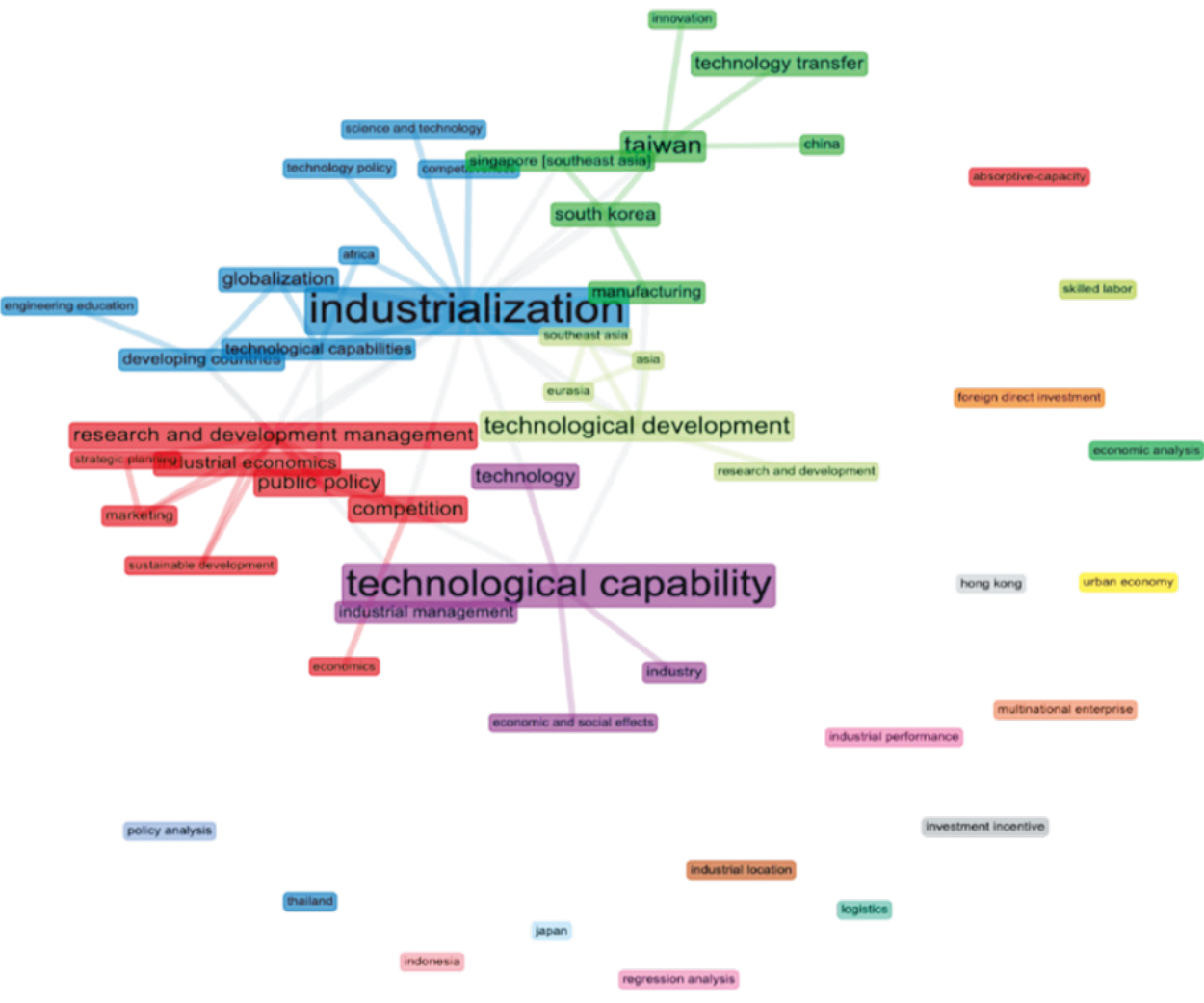

Figura 3. Gráfico de co-ocurrencias basado en al EB.

Fuente: elaboración propia con base en Aria y Cuccurullo (2017).

Finalmente, se destacan los siguientes hallazgos: i) se evidencian algunas revistas sobresalientes dentro de las publicaciones (International Journal of Technology Management (4), World Development (4), African Journal of Science Technology Innovation and Development (2), European Planning Studies (2), Journal of the Asia Pacific Economy (2), Technological Forecasting and Social Change (2) y Technovation (2); ii) el número de citaciones es bajo frente a resultados obtenidos en otras áreas de conocimiento con las ecuaciones de búsqueda ampliadas; iii) frente a la producción científica según el país sobresale Estados Unidos con seis publicaciones y Brasil, Canadá, China y Reino Unido con cuatro publicaciones cada uno.

\section{Análisis cualitativo}

Es pertinente hacer dos aclaraciones frente al análisis detallado de las publicaciones que se presenta a continuación. 
En primer lugar, los trabajos de Antrim (2005), Amsden y Hikino (2006) y López (2010) no se contemplan en esta sección al no estar asociados estrechamente con la pregunta de investigación propuesta, a pesar de que fueron identificadas por la EB. En segundo lugar, con el fin de potenciar la revisión sistemática de literatura, se incluyeron algunas referencias relevantes citadas en las publicaciones mencionadas, tales como Fransman y King (1984), Alam (1985), Kim y Kim (1985), Pack y Westphal (1986), Reddy y Zhao (1990), Bell y Pavitt (1992), Kogut y Zander (1992), Dutrénit y Capdevielle (1993), Cusumano y Elenkov (1994), Freeman (1994), Villavicencio y Arvanitis (1994), Biggs et al. (1995), Brown y Domínguez (2004), Dutrénit (2004), Coombs y Bierly (2006), Dutrénit (2007), Pérez-Escatel y Pérez (2009), Hansen y Ockwell (2014) y Kiamehr (2017).

El estudio realizado por Lall (1992) es, en esencia, el primer intento por revisar las implicaciones para la estrategia industrial que se derivan de la investigación reciente en torno a las capacidades tecnológicas. Tal como se precisa en el documento, la literatura reciente -con trabajos como los de Wong y Mathews (2005), Hsu et al. (2008), Zhou et al. (2009), Lee et al. (2010), Chang (2012), Peerally y Cantwell (2012), More y Jain (2013), Figueiredo (2014), Lavarello et al. (2017), Arya y Bhaduri (2018), Kale
(2019) y Jun y Rowley (2019) - ha puesto en el centro del debate el impacto que tiene el desarrollo de capacidades tecnológicas en las empresas, las industrias y los países ${ }^{3}$. En este sentido, Lall (1992) concibe las capacidades tecnológicas desde el nivel de la firma hacia el nivel país mediante tres categorías: i) inversión física, ii) capital humano y iii) esfuerzos tecnológicos.

Desde hace algunas décadas la evidencia empírica demuestra que la tecnología en el contexto de los mercados internacionales difiere en sus niveles entre economías industrializadas y no industrializadas; sobre el particular, Adei (1990) estudia la experiencia de "Bonsa Tire Company", empresa ghanesa que no logró enfocar sus esfuerzos en el fortalecimiento de la capacidad tecnológica dentro de la compañía, y precisa que la debilidad en la capacidad tecnológica de la empresa ha sido identificada como un factor importante que explica el bajo rendimiento.

El trabajo reciente de Arvanitis et al. (2014) sigue esta base de análisis para economías emergentes en contraste con economías industrializadas; es una reflexión teórica basada en estudios empíricos llevados a cabo en América Latina y China para identificar y caracterizar el aprendizaje tecnológico de las empresas, distanciándose de las perspectivas tradicionales que se enfo-

3 Es relevante mencionar que desde la literatura económica clásica y neoclásica se pone de manifiesto que la tecnología está disponible en todo momento y lugar para las empresas que compiten en un escenario de competencia perfecta. 
can en la identificación de actividades tecnológicas internas y externas, así como el conocimiento calificado de las "técnicas" que acompañan estas actividades. Los autores se centran en las decisiones económicas que determinan estas actividades y la aparición de empresas innovadoras en las economías emergentes como producto de dichas decisiones, focalizan su atención en organizar las zonas densas de aprendizaje colectivo en las empresas, de tal forma que faciliten el crecimiento de las empresas industriales en torno a distritos o grupos de empresas que conforman clústeres y desarrollen vínculos útiles para alimentar su capacidad productiva y beneficiarse de externalidades positivas.

Las conclusiones establecidas por Villavicencio y Arvanitis (1994) se hacen eco de esta afirmación, en las cuales los autores precisan que “...) algunas de estas actividades están diseñadas para reforzar el potencial humano de la empresa para controlar sus equipos y esto se traduce en una consolidación de la investigación de la información, ingeniería, I+D” (2014, p. 505). De hecho, los estudios de caso demuestran que una empresa compuesta inicialmente por personal relativamente poco capacitado podría ser particularmente eficiente e innovadora debido a la construcción colectiva de habilidades (Villavicencio \& Arvanitis, 1994).
A partir de estas reflexiones es posible afirmar que, en la literatura sobre el aprendizaje tecnológico en empresas, industrias y clústeres en las economías industrializadas: “(...) la mayor parte se ha centrado en cuestiones relacionadas con el cambio a lo largo del tiempo, caminos de cambio técnico, trayectorias de acumulación de capacidades y las fuerzas que dan forma a estas tendencias" (Bell, 2006, p. 25). Tal como indica Oyelaran-Oyeyinka (2003), los clústeres se caracterizan por dos elementos dinámicos: las tasas y los tipos de aprendizaje tecnológico, y la naturaleza e intensidad del trabajo en red; por lo cual puede esperarse que exista un correlación significativa entre las capacidades tecnológicas en el ámbito de la firma y las redes de conocimiento que permiten la construcción del capital social en el marco de las industrias, clústeres o distritos industriales. De esta manera, el estudio en mención busca examinar la intensidad de las redes y sus connotaciones (e.g. subcontratación de fuerza de trabajo) como bases del conocimiento tecnológico en el marco de clústeres conformados en Nigeria, y encuentra que una mayor subcontratación se traduce en vínculos más fuertes entre empresas que pertenecen al clúster; por consiguiente, es de suponer que la subcontratación es un tipo importante de vinculación contractual en el que la colaboración entre las empresas se ve incentivada con el propósito de reducir los costos de transacción. 
Por otro lado, el aprendizaje como determinante del proceso de acumulación de capacidades tecnológicas también es ampliamente abordado por Kiamehr (2017), el cual busca investigar la acumulación de capacidades tecnológicas en proveedores de bienes de capital complejos para el caso de una empresa iraní de generación hidroeléctrica. Los hallazgos sugieren que el proceso de acumulación de capacidades tecnológicas no inicia desde las primeras etapas del ciclo de vida del producto; de hecho, las capacidades tecnológicas para esta empresa iraní se adquirieren mediante un movimiento no lineal dentro de las etapas de diseño e instalación de bienes de capital complejos en proyectos: comenzando desde la etapa intermedia (ingeniería y realización de bienes complejos en proyectos), pasando a la última etapa (operación y resolución de problemas de bienes complejos), y finalmente regresando a la primera etapa (concepción y diseño de bienes complejos). De esta forma, el estudio sugiere que el "aprendizaje por proyectos" es un importante determinante de la acumulación de capacidades tecnológicas.

Por su parte, Gammeltoft (2003), desde el marco de la organización industrial, expone la idea que se basa en el reconocimiento de que las economías se desarrollan gradualmente a través de la expansión acumulativa tanto de la capacidad de producción como de las estructuras organizativas o institucionales. Al respecto, Gammeltoft (2004) se enfoca en discutir el concepto de capacidades tecnológicas en el marco de las empresas locales de la industria tecnológica de Indonesia, para lo cual establece un modelo de desarrollo de capacidades en la empresa en el que se destacan cuatro tipos de capacidades: i) proceso adquisitivo, ii) proceso operativo, iii) cambio de proceso y iv) cambio de producto. Desde esta perspectiva, el articulo contribuye a esclarecer el estado y el desarrollo de la industria con fundamento en el incremento de capacidades tecnológicas, e identifica tres tipos de empresas: i) "empresas intrínsecas", teniendo Indonesia como su base de operaciones; ii) empresas "extrínsecas", que no tienen a Indonesia como su base de operaciones, y iii) "empresas orientadas al Estado", que son empresas estatales o empresas privadas impulsadas por la contratación pública. Al contrario de lo esperado, las capacidades operativas de los fabricantes de productos intrínsecos aparecieron bastante altas, lo cual tiene explicación debido a que al ser más autosuficientes y autónomas, estas empresas tienen capacidades adquisitivas y de cambio relativamente altas. Por otro lado, el autor indica que "(...) las empresas extranjeras que aplican tecnologías avanzadas de hardware y software y, por lo general, operan con una mayor utilización de la capacidad pueden producir de manera más eficiente" (Gammeltoft, 2004, p. 65). De manera similar, Liu (1998) analiza el éxito industrial de Taiwán identificando como elementos claves la capacidad de aprendizaje y la acumulación de capital humano orien- 
tado por la sinergia entre los mercados y el Estado. Adicionalmente, Bowonder (1998) defiende el papel de la globalización y la liberalización de los mercados como promotor de la competencia y el desarrollo de capacidades tecnológicas en India con el fin de potenciar la infraestructura tecnológica existente.

Queda claro que la dinámica de acumulación de capacidades tecnológicas en los países subdesarrollados difiere sustancialmente de la dinámica de las economías industrializadas y desarrolladas. Hace algunas décadas, Fransman y King (1984) argumentaron que el tercer mundo posee capacidades tecnológicas débiles y que, por lo tanto, el enfoque debía estar en la importación de tecnologías extranjeras, más que en el desarrollo o acumulación de las capacidades tecnológicas locales. Esta afirmación es de importancia en el presente, dado que diversos estudios demuestran que los términos de intercambio entre economías desarrolladas y subdesarrolladas profundizan y acentúan el intercambio desigual; sin embargo, el enfoque de importación de capacidades tecnológicas es un frente de investigación pertinente, donde se entiende que la eficiencia productiva también puede explicarse en cierta medida por la importación de conocimiento, $i$. e. acumulación de capacidades tecnológicas a través de mecanismos alternativos para adquirir tecnología extranjera, a medida que la industrialización avanza en los países en desarrollo.
De manera complementaria, Kim (1999) destaca que los proveedores extranjeros y los fabricantes de equipos originales a menudo transfieren conocimientos críticos a los productores para garantizar que los productos cumplan con las especificaciones técnicas de los compradores. En otras palabras: “(...) la información impresa, como catálogos de ventas, planos, especificaciones técnicas, publicaciones comerciales y otras publicaciones, junto con la observación de plantas extranjeras, sirven como importantes fuentes informales de nuevos conocimientos para empresas en países en desarrollo" (Kim \& Kim, 1985, p. 314). La fuga de cerebros hacia la economía doméstica y el retorno de profesionales formados en el extranjero probablemente logren aumentar la capacidad de aprendizaje tecnológico en las empresas de economías emergentes; adicionalmente, si las empresas de las economías en vía de desarrollo tienen capacidad de absorción, pueden adquirir efectivamente tecnología extranjera de manera informal sin ningún costo de transacción (Kim, 1999).

Adicionalmente, es innegable que la transferencia de tecnología extranjera a economías emergentes o en vías de desarrollo debe también incorporar un esfuerzo por comprender los procesos de aprendizaje que de allí se derivan, para lograr acumular capacidades tecnológicas, de formación y desarrollo industrial. En general, los estudios demuestran que las economías emergentes pasan por alto las características 
y condiciones específicas de las empresas. En este sentido, los trabajos de Iguchi (2008), Moreira (2008), Poon (2010), Lorentzen et al. (2011), Chen (2013) y Myint et al. (2015) analizan la posibilidad de generar encadenamientos hacia atrás y hacia adelante por parte de oferentes locales con corporaciones transnacionales por medio de inversión extranjera directa y transferencia de tecnología, como forma de promover la industrialización y la acumulación de capacidades tecnológicas

En este sentido, Hansen y Ockwell (2014), y de manera similar Shi (2005), en lo relacionado con el papel de la inversión extranjera directa como motor de industrialización en China, contribuyen a llenar este vacío al utilizar en profundidad datos cualitativos de la empresa para analizar hasta qué punto el uso de diferentes mecanismos de aprendizaje puede explicar las diferencias en el proceso de acumulación de capacidades tecnológicas, para lo cual centran el análisis en ocho empresas de la industria de equipos de energía de biomasa en Malasia durante el período 1970-2011; entre sus principales conclusiones se destaca que las empresas que confían en una combinación de aprendizaje de socios tecnológicos extranjeros y el aprendizaje interno mediante la experimentación planificada, logran el mayor progreso en términos de capacidad tecnológica, y aquellas empresas que buscan aprender de socios extran- jeros de manera proactiva alcanzan niveles innovadores básicos de capacidades tecnológicas.

Por su parte, Madanmohan et al. (2004) investigan los factores que afectan la capacidad de las empresas en los países en desarrollo para cultivar sus capacidades tecnológicas a través de la tecnología importada, destacando el rol del Gobierno y la infraestructura tecnológica nacional como factores externos que afectan las capacidades tecnológicas de la empresa. En su investigación, en concordancia con Sussangkarn y Manupipatpong (2015), los datos recopilados de las empresas manufactureras indias e indonesias revelan que la inversión en $\mathrm{I}+\mathrm{D}$, la disponibilidad de personal técnico, los canales de transferencia, la participación del Gobierno y la cultura de aprendizaje de la empresa contribuyen significativamente al proceso de capacidad tecnológica.

Diversos autores han centrado su atención en analizar estos factores internos y su relación con el desarrollo de capacidades tecnológicas; se destacan los trabajos de Kogut y Zander (1992), Cusumano y Elenkov (1994) y Reddy y Zhao (1990), los cuales resaltan la idea de que la capacidad de las empresas en las economías en vías de desarrollo para absorber la tecnología importada depende de sus capacidades estratégicas internas. En cuanto a los factores externos, Madanmohan et al. (2004) identifican al Gobierno (cuya participación va desde el apoyo activo 
en forma de subsidios, beneficios tributarios y métodos más indirectos hasta las regulaciones gubernamentales que tienen una influencia significativa en el cambio tecnológico) y la infraestructura tecnológica nacional como factores determinantes del desarrollo tecnológico, lo cual concuerda con el trabajo de Alam (1985).

En relación con los factores externos mencionados, Fessehaie (2013) afirma que el Gobierno es un agente de importancia preponderante como dinamizador del proceso de acumulación de capacidades tecnológicas; puntualmente, investiga la transferencia de la cadena de suministro local de Zambia al sector de la minería del cobre, en el marco del Programa de Ajuste Estructural durante la década de 1990, cuyas políticas adoptadas permitieron la llegada de inversión extranjera directa, necesaria para incentivar la acumulación de capacidades tecnológicas; no obstante, el desmonte de las políticas industriales implicó que las empresas ya no recibieran apoyo para desarrollar capacidades tecnológicas y acceder a habilidades y capital, lo cual se tradujo en un proceso de privatización en donde la gobernanza de la cadena de valor se ve afectada de forma negativa en el propósito de apoyar la actualización tecnológica de las empresas proveedoras de suministros.
En síntesis, puede afirmarse que el proceso de acumulación de capacidades tecnológicas puede analizarse desde diferentes perspectivas y categorías; así, Kim (1999) desarrolla un modelo integrador para examinar el proceso de aprendizaje tecnológico en la empresa a partir de cinco categorías de análisis: i) trayectoria tecnológica, ii) capacidad de absorción, iii) transferencia de tecnología iv) construcción de crisis y v) proceso de aprendizaje dinámico; todas importantes al analizar el proceso de creación de capacidades tecnológicas para la industrialización.

Ha de entenderse que el proceso de acumulación de capacidades tecnológicas se traduce en innovaciones de productos y procesos desde la perspectiva de cambio tecnológico continuo, una idea desarrollada por Pack y Westphal (1986), que considera el cambio tecnológico como el corazón del proceso de industrialización dinámica ${ }^{4}$. Así, la innovación resulta ser un componente importante de análisis, ya que se entiende como una cuestión de supervivencia en el marco de la globalización; de esta forma, la literatura que relaciona las capacidades tecnológicas y la innovación es amplia y cuenta con diversas perspectivas y enfoques teóricos, como el caso de Santos et al. (2015), quienes realizan una revisión bibliométrica de literatura en la que buscan mapear los autores, las instituciones, las revistas y la evolución de la producción científica

4 Es importante mencionar que el autor presenta como ejemplo la experiencia de Corea del Sur, país que ha logrado percibir un elevado crecimiento económico en el marco de la acumulación y creación de capacidades tecnológicas 
en torno a la innovación y las capacidades tecnológicas, delimitando un estado del tema adecuado que ubica a este campo de estudio como emergente en la investigación de la competitividad de las organizaciones.

Una perspectiva alternativa es entregada por Dahlman et al. (1987), quienes afirman que el centro del desarrollo tecnológico es adquirir las capacidades necesarias para una producción e inversión eficientes, considerando que los países y firmas que han invertido demasiado esfuerzo en lograr la autosuficiencia tecnológica han experimentado, de hecho, escenarios de baja productividad, y concluyen que es mucho más eficiente, en el sentido económico, combinar componentes tecnológicos locales y extranjeros, y hacerlo de una manera que desarrolle progresivamente las capacidades locales en áreas donde puedan ser más eficientes. Por tanto, la adquisición de la capacidad tecnológica no proviene únicamente de la experiencia, sino también de esfuerzos por monitorear y realizar seguimiento de los desarrollos en todo el mundo, acumular habilidades y aumentar la capacidad de responder ante los desafíos de la competencia en los mercados internacionales. Finalmente, el entorno económico, a través de su influencia en los esfuerzos tecnológicos de las empresas, desempeña un rol muy importante para determinar la productividad de los recursos.
A partir de lo anterior, a través del proceso de acumulación de capacidades tecnológicas a escala microeconómica, las economías de escala hacen presencia no solo en el marco de los procesos productivos, sino también en el proceso de creación de capital social entre empresas de un sector o un grupo de sectores, lo que a medio plazo llega a constituirse como factor determinante de la competitividad (Jonker et al., 2006). En el estudio mencionado, los autores desarrollan algunas medidas cuantitativas respecto a variables como el esfuerzo tecnológico referido a la máquina, capacidades tecnológicas y desempeño económico para la industria del papel en Java Occidental en Indonesia para llegar a concluir que es factible operacionalizar y medir los esfuerzos tecnológicos, las capacidades tecnológicas y el desempeño económico referido a la máquina, aunque no encuentran correlaciones positivas y significativas entre los diversos indicadores del esfuerzo tecnológico y las capacidades tecnológicas; no obstante, estos resultados se justifican desde Biggs et al. (1995), quienes precisan que para la manufactura africana, el nivel general de los esfuerzos de aprendizaje y los vínculos en los países en desarrollo es tan bajo que no se puede esperar mucha variación en los efectos de los esfuerzos de aprendizaje.

Por otra parte, si bien se entiende que existe una relación no explícita entre la acumulación de capacidades tecnológicas y la competitividad, Pérez-Escatel 
y Pérez (2009) plantean una relación implícita que resalta la importancia de analizar el comportamiento tecnológico de las empresas manufactureras a partir de la apertura comercial e identificar los efectos en la adquisición de capacidades tecnológicas en el marco de las firmas mexicanas, basándose en el supuesto de que las ventajas competitivas se manifiestan a través de la acumulación o adquisición de capacidades tecnológicas. En este sentido, Dutrénit y Capdevielle (1993) expresan la idea de que la acumulación de capacidades tecnológicas surge a partir de un nivel mínimo de conocimiento tecnológico, generalmente en el escenario en donde las empresas son jóvenes y se centran en aprender de la experiencia, por lo que luego de un determinado tiempo, es probable que surjan nuevas actividades productivas que implican la acumulación de capacidades tecnológicas para llevar a cabo procesos productivos innovadores o actividades de innovación; sin embargo, Dutrénit (2004) indica que no existe una progresión lineal simple desde la etapa inicial de acumulación de los niveles mínimos de capacidad innovadora hasta la gestión del conocimiento como un activo estratégico y el despliegue de capacidades básicas. Por el contrario, el proceso de transición es complejo y si bien las empresas hacen esa transición, tienen que crear reservas de conocimiento más profundas y amplias y desarrollar nuevos tipos de gestión del conocimiento5.

De manera complementaria, Coombs y Bierly (2006) precisan que la relación existente entre las capacidades tecnológicas y el desempeño o rendimiento de la empresa es más compleja de lo que puede suponerse, teniendo en cuenta que la literatura que provee evidencia empírica de esta relación subyacente es escasa; por esta razón, los autores buscan dimensionar e ilustrar la complejidad teórica y empírica de la misma y explicar por qué el uso de diversas medidas puede conducir a resultados muy diferentes. A tal fin, analizan la relación capacidad-desempeño tecnológico en 201 empresas manufactureras públicas de gran tamaño en los Estados Unidos, y encuentran que utilizar el número total de patentes y el gasto en I+D no son medidas válidas de las capacidades tecnológicas de una firma.

Cabe precisar que la mejora en el rendimiento de las firmas a través de la acumulación de capacidades tecnológicas probablemente debe traducirse en una mejora de la competitividad del país. En este sentido, Freeman (1994)

$5 \quad$ Esta reflexión se refuerza en Dutrénit (2007), quien hace énfasis en el proceso de transición desde el proceso de desarrollo de capacidades tecnológicas hacia el desarrollo de capacidades estratégicas con el propósito de acercarse a la frontera tecnológica. De igual forma, el autor precisa que la literatura basada en el vínculo entre el aprendizaje y la acumulación de capacidades tecnológicas se ha centrado principalmente en analizar los procesos de aprendizaje involucrados en la construcción gradual de una base mínima de conocimiento tecnológico para poder llevar a cabo actividades innovadoras (en la misma línea de Bell \& Pavitt, 1992). Cabe destacar que en esta descripción se concibe que las capacidades tecnológicas (habilidades y conocimiento tecnológico) serán necesarias para desarrollar, producir y vender nuevos productos, además de lograr crear nuevas tecnologías que sirvan a los propósitos señalados. 
destaca el rol que desempeña el cambio técnico como determinante de las innovaciones, su difusión en la firma y en la industria, esperando entonces que la competitividad de un país también esté determinada por el proceso de cambio técnico. En esta línea, con la intención de medir cuantitativamente la relación existente entre la acumulación de capacidades tecnológicas y la competitividad, Pérez-Escatel y Pérez (2009) siguen la metodología de Brown y Domínguez (2004), en la cual construyen un índice de capacidades tecnológicas con base en las consideraciones teóricas y prácticas de Lall (1992) y Bell y Pavitt (1992).

Cabe precisar que la obra de Biggs et al. (1995) puede considerarse como uno de los primeros intentos sistemáticos por evaluar la relación existente entre las capacidades tecnológicas y la productividad de las firmas al utilizar datos primarios de dos encuestas aplicadas en empresas manufactureras en Ghana, Kenya y Zimbabwe. Este trabajo se centra en aspectos generales relacionados con determinantes de la productividad manufacturera, los niveles de dotación de capacidades tecnológicas, la naturaleza específica de los esfuerzos tecnológicos emprendidos por empresas manufactureras y las limitaciones que enfrentan para mejorar tales esfuerzos. De esta forma, busca comprender en detalle el vínculo que existe entre las capacidades tecnológicas, la productividad técnica y el crecimiento de la firma, y se plantea la posibilidad de que exista un canal de transmisión de efectos entre la acumulación de las capacidades tecnológicas y el crecimiento económico.

Finalmente, cabe destacar los aportes de Rasiah (2004), quien construye un análisis amplio centrándose en las experiencias de economías asiáticas, africanas y latinoamericanas, empleando novedosas técnicas para las capacidades tecnológicas y el desempeño económico para países con diferentes niveles de industrialización: Brasil, Costa Rica, Indonesia, Kenia, Malasia, Sudáfrica África y Uganda. El estudio en mención es especialmente riguroso en el marco conceptual empleado, así como en la cobertura de la literatura abordada, lo que lo ha convertido en una de las publicaciones de mayor consulta respecto a la relación existente entre el desempeño económico y las capacidades tecnológicas en el sentido propuesto por Sanjaya Lall, para llevar a cabo una serie de ensayos en los que se profundiza en el análisis del rol que cumplen las firmas extranjeras en la mejora de las capacidades tecnológicas y en la competitividad.

\section{DISCUSIÓN Y REFLEXIÓN TEÓRICA}

Una vez hecha la revisión sistemática de literatura, se resalta que el estudio de las capacidades tecnológicas en el ámbito de la firma -y en el nacional- es un área de interés para la economía y la gestión de organizaciones, dado que propende a identificar determinantes claros para 
la acumulación de dichas capacidades, con el fin de potenciar la competitividad industrial y generar un proceso de retroalimentación continua, en el que los estudios empíricos revisados y de reflexión teórica permiten vislumbrar un panorama más claro sobre el concepto desarrollado por Sanjaya Lall a lo largo de su recorrido académico.
A partir de la revisión, basados especialmente en los aportes de Kim (1999) y Madanmohan et al. (2004), se construye el siguiente esquema sintético que vislumbra la dinámica de las capacidades tecnológicas a la luz de sus principales determinantes, sus condiciones necesarias y sus mediadores:

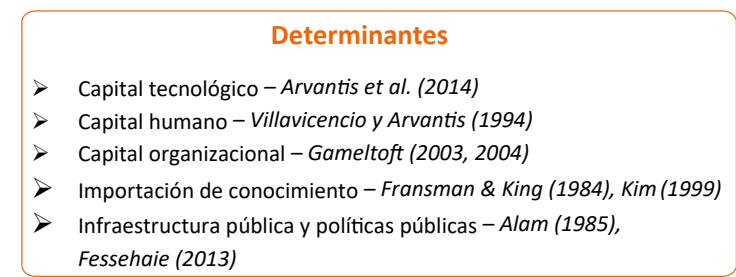

Fessehaie (2013)
Economía de mercado + Instituciones

\section{Capacidades Tecnológicas}

\section{Mediadores}

Externalidades positivas - Kim \& Kim (1985)

Interacción tipos de capital (tecnológico, humano, organizacional) - Pérez,_Escatel \& Pérez (2009)

Aprendizaje colaborativo - Kiamehr (2017), Hansen y Okwell (2014), Dahlman et al. (1987)

Innovación - Santos et al. (2015)

Figura 4. Dinámica de las capacidades tecnológicas

Fuente: elaboración propia.

El esquema construido permite dimensionar la relación existente entre los determinantes de la acumulación de las capacidades tecnológicas de las empresas en el marco de una economía de mercado e instituciones formales que se orienten al desarrollo productivo, cuyos efectos mediadores potencian dichas relaciones. A partir de lo anterior, no se puede perder de vista que la razón por la cual deben existir estos efectos mediadores obedece al hecho de que el proceso de acumulación de capacidades tecnológicas es un pro- 
ceso dinámico no lineal $\mathrm{y}$, por ende, requiere de un esfuerzo por identificar factores que permitan la sinergia entre determinantes, condiciones necesarias y acumulación de capacidades.

Entre las condiciones necesarias (mínimas) para la acumulación de capacidades tecnológicas se destaca la consecución de economías de escala que sean un reflejo de la eficiencia productiva y que permitan también la creación de capital social entre empresas de un sector o un grupo de sectores (Jonker et al., 2006). Sin embargo, para lograr elevar los niveles de producción y reducir continuamente los costos medios de producción en el tiempo (economías de escala) se requiere de un mínimo de conocimiento tecnológico base para aprender de la experiencia y permitir el surgimiento de nuevas actividades productivas que impliquen la acumulación de capacidades tecnológicas para llevar a cabo procesos productivos innovadores (Dutrénit \& Capdevielle, 1993). De igual forma, el cambio técnico como condición necesaria es un mecanismo de difusión de habilidades de conocimiento en la firma y en la industria, lo cual brinda oportunidades inigualables de aprendizaje derivado de la experiencia productiva (Freeman, 1994).

Ahora bien, el aprendizaje colaborativo como elemento mediador entre las condiciones necesarias y los determinantes de la acumulación de capacidades tecnológicas, tiene diferentes connotaciones: en primer lugar, el aprendizaje que se logra a través del trabajo colaborativo en el marco de la formulación y ejecución de proyectos que van desde las etapas de diseño, ingeniería y operación y resolución de problemas (Kiamehr, 2017); en segundo lugar, el aprendizaje también puede conseguirse a través de importaciones (Dahlman et al., 1987) si se considera que es mucho más eficiente, en el sentido económico, combinar componentes tecnológicos locales y extranjeros, y hacerlo de una manera que desarrolle progresivamente las capacidades locales en áreas donde puedan ser más eficaces; en tercer lugar, la acumulación de capacidades tecnológicas no proviene únicamente de la experiencia, proviene también de esfuerzos por monitorear y realizar seguimiento de los desarrollos en todo el mundo, los cuales pueden implicar revisión de información impresa (i. e. especificaciones técnicas, publicaciones comerciales y otras publicaciones) y un proceso de observación de plantas extranjeras, lo cual sirve como importante fuente de nuevos conocimientos (Kim \& Kim, 1985); en este sentido, una postura intermedia es presentada por Hansen y Ockwell (2014) al precisar que las empresas que confían en una combinación de aprendizaje de socios tecnológicos extranjeros y el aprendizaje interno, mediante la experiencia logran el mayor progreso en términos de capacidad tecnológica.

El proceso de vigilancia tecnológica también depende de los resultados del mercado internacional y del ajuste 
estratégico temporal de las empresas con el entorno externo; se trata de un proceso de sincronización temporal de los parámetros de internacionalización como una estrategia de adaptación a las dinámicas globales (Popli et al., 2017). En este sentido, Pérez (2020), mediante un análisis panel con efectos temporales encuentra que las importaciones de insumos tecnológicos y de productos de alta complejidad constituyen un factor que impulsa la capacidad tecnológica en México, lo que valida la idea de que los componentes mediadores preparan el escenario propicio para explorar los canales que permiten acumular efectivamente las capacidades tecnológicas.

Cabe destacar que el proceso de diversificación tecnológica en las capacidades tecnológicas desempeña un papel determinante para reforzar la ventaja competitiva. Al respecto, Kook et al. (2017), tras analizar la evolución temporal de la diversificación tecnológica en Corea del Sur, recomiendan que la empresa debe concentrarse/especializarse en una tecnología específica para reforzar la ventaja competitiva y posteriormente explotar la diversificación tecnológica; así pues, esta perspectiva abre un enfoque de análisis temporal relacionado con el ajuste dinámico de las capacidades tecnológicas en el tiempo y su resultado en la generación de externalidades positivas producto de su diversificación.

Con la aparición de externalidades positivas como resultado de los procesos mediadores ya descritos, la innovación es un componente importante de análisis, ya que se entiende como una cuestión de supervivencia en el marco de la globalización (Santos et al., 2015), la cual va de la mano de la interacción de los diferentes tipos de capital dentro de las firmas (tecnológico, humano, organizacional, entre otros) para potenciar la inserción de estas en mercados internacionales de manera efectiva (Pérez-Escatel \& Pérez, 2009).

Finalmente, se destaca el capital organizacional como uno de los principales determinantes de la acumulación de capacidades tecnológicas, el cual logra dinamizar la expansión acumulativa tanto de la capacidad de producción como de las estructuras organizativas o institucionales (Gammeltoft, 2003, 2004), teniendo en cuenta que las actividades organizativas desempeñan un rol importante para alcanzar el conocimiento calificado de las "técnicas" que acompañan estas actividades. Además, el conocimiento se potencia aún más en la medida en que el trabajo colaborativo se traduce en un proceso de aprendizaje técnico; la literatura indica que buena parte del esfuerzo organizativo está diseñado para reforzar el potencial humano de la empresa para controlar sus equipos, lo que se traduce en una consolidación de la investigación de la información, ingeniería, $\mathrm{I}+\mathrm{D} \mathrm{y}$, por ende, en la acumulación de capital humano (Villavicencio \& Arvanitis, 1994) y capital tecnológico (Arvanitis et al., 2014). De manera transversal, el Gobierno desempeña un papel relevante a través de una sólida infraestructura 
institucional y la infraestructura tecnológica nacional, como factores determinantes del desarrollo tecnológico en la firma, los cuales van desde el apoyo activo en forma de subsidios, beneficios tributarios y métodos más indirectos, hasta las regulaciones gubernamentales que tienen una influencia significativa en el cambio tecnológico (Alam, 1985).

\section{CONCLUSIONES}

En esta reflexión teórica se observa que el proceso de acumulación de capacidades tecnológicas en la firma está permeado por factores contemplados en los niveles meso y macro de la competitividad (Chica, 2007a); es un proceso de retroalimentación continua que busca la generación de valor agregado en los bienes y servicios ofrecidos por las organizaciones, en el que las exportaciones de mediana y alta tecnología son un elemento clave del crecimiento y el desarrollo económico, según los planteamientos de Sanjaya Lall. En este sentido, su enfoque de capacidades tecnológicas como determinante fundamental de la competitividad industrial permite conectar el análisis económico de este fenómeno con la dinámica estratégica de la organización, al identificar determinantes claves como la acumulación e interacción de los diferentes tipos de capital, la importación de conocimiento y la infraestructura y políticas públicas definidas por los gobiernos. De esta manera, se obtiene una mirada más amplia frente a la interacción de los niveles de la competitividad propuestos por Chica (2007a), los cuales van en consonancia con el proceso de causación acumulativa o círculo virtuoso desarrollado por Kaldor (1961).

Por otra parte, la revisión sistemática de literatura permitió conocer un área de investigación relevante para la organización industrial y la estrategia empresarial, con una rica reflexión teórica acompañada de diversos trabajos empíricos que facilitaron la construcción del esquema sintético propuesto, siendo fundamentales tres publicaciones con un desarrollo conceptual y analítico de soporte: Kim (1999), Madanmohan et al. (2004) y Pérez-Escatel y Pérez (2009), los cuales llevaron a identificar los elementos esenciales de la acumulación de capacidades tecnológicas: las condiciones necesarias, los mediadores y los determinantes resaltados en la sección anterior.

A partir de este ejercicio de revisión, se proponen tres líneas futuras de investigación: i) teniendo en cuenta que la mayoría de los trabajos que están en consonancia con Lall pretenden identificar la dinámica de las capacidades tecnológicas en países emergentes, sería interesante llevar a cabo estudios comparativos entre firmas de economías desarrolladas y economías emergentes, que tuvieran en cuenta el esquema sintético construido para proponer políticas públicas y estrategias empresariales; para ello, los modelos construidos por Pérez-Escatel y Pérez 
(2009) representan un insumo valioso; ii) considerando que las firmas se desempeñan en escenarios de competencia imperfecta, se podrían incluir variables asociadas a fallos del mercado (e. g. asimetrías de información dentro de las organizaciones) y fallos del Estado (e.g. distorsión en estructuras de incentivos) con el fin de tener un panorama acorde con el nivel meso de la competitividad descrito anteriormente, y iii) incluir dentro de los estudios de capacidades tecnológicas de la firma, variables macrofundamentales como las descritas por Chica (2007a), que capturen la dinámica de estabilidad, crecimiento $\mathrm{y}$ desarrollo de las economías en las que se encuentra la firma.

Otra línea de investigación que emerge del estudio es la orientada a indagar las interacciones entre las capacidades tecnológicas desde su perspectiva económica y los recursos y capacidades desde la literatura estratégica y administrativa, de manera que los aportes de la gestión del conocimiento (Gaviria-Marín et al., 2019; Oliva et al., 2019; Martins et al., 2020), del aprendizaje organizacional (Hansen \& Lema, 2019; Ipek, 2019; Zuo et al., 2019) y del capital intelectual (Ahmad \& Bin Mohammad, 2019; Martín et al., 2019), junto con el enfoque de las capacidades dinámicas (Teece \& Pisano, 2003; Carattoli, 2013; Albort-Morant et al., 2018), se complementen y produzcan efectos sinérgicos. Incorporar a la lectura económica de las capacidades tecnológicas aspectos como la gestión y métricas de los recursos/activos intangibles de la firma, los escenarios, ambientes y procesos de aprendizaje individual, colectivo, organizativo e interorganizativo, y el sistema de generación, difusión, aplicación, institucionalización y explotación del conocimiento, representa una oportunidad para analizar y conseguir una mayor comprensión de la temática, así como para mejorar las decisiones y acciones al respecto, a partir del trabajo interdisciplinar.

\section{AGR ADECIMIENTOS}

Los autores agradecemos los comentarios de los evaluadores anónimos de la Revista.

\section{CONTRIBUCIÓN DE LOS AUTORES}

Los autores participaron de manera conjunta a lo largo de la construcción del artículo.

\section{DECLARACIÓN DE CONFLICTO DE INTERESES}

Los autores declaran que no existe conflicto de intereses.

\section{FINANCIAMIENTO}

Este trabajo no contó con ningún tipo de financiamiento institucional, fue realizado con recursos humanos de los autores. 


\section{REFERENCIAS}

[1] Adei, S. (1990). Technological Capacity and Aborted Industrialization in Ghana: The Case of Bonsa Tyre Company. World Development, 18(11), 1501-1511. https://doi.org/10.1016/0305-750X(90)90039-Z

[2] Ahmad, R., \& Bin Mohammad, H. (2019). Exploring the Relationship of Intellectual Capital, Board Characteristics and Business Performance: A Literature Review. Pakistan Journal of Humanities and Social Sciences, 7(2), 185-202. https://ssrn. com/abstract $=3431798$

[3] Alam, G. (1985). India's Technology Policy and Its Influence on Technology Imports and Technology Development. Economic and Political Weekly, 20(45, 46, 47), 2073-2080.

[4] Albort-Morant, G., Leal-Rodríguez, A., Fernández-Rodríguez, V. \& Ariza-Montes, A. (2018). Assessing the Origins, Evolution and Prospects of the Literature on Dynamic Capabilities: A Bibliometric Analysis. European Research on Management and Business Economics, 24, 42-52. http://dx.doi.org/10.1016/j. iedeen.2017.06.004

[5] Al Jinini, D. K., Dahiyat, S. E., \& Bontis, N. (2019). Intellectual Capital, Entrepreneurial Orientation, and Technical Innovation in Small and Medium Sized Enterprises. Knowledge and Process Management, 26(2), 69-85. https://doi.org/10.1002/kpm.1593

[6] Ambrosini, V., Bowman, C. \& Collier, N. (2009). Dynamic Capabilities: An Exploration of How Firms Renew their Resource Base. British Journal of Management, 20(1), S9-S24. https://doi.org/10.1111/j.1467-8551.2008.00610.x

[7] Amsden, A.H. \& Hikino, Takashi. (2006). Economic Nationalism and Income Distribution in Late Industrializing Countries. https://www.researchgate.net/ publication/295756539_Economic_nationalism_and_income_distribution_in_ late_industrializing_countries

[8] Antrim, C. L. (2005, September). What Was Old Is New Again: Economic Potential of Deep Ocean Minerals the Second Time Around. In Proceedings of OCEANS 2005 MTS/IEEE (pp. 1311-1318). IEEE. https://oi.org/10.1109/ OCEANS.2005.1639936

[9] Aria, M., \& Cuccurullo, C. (2017). Bibliometrix: An R-Tool for Comprehensive Science Mapping Analysis. Journal of Informetrics, 11(4), 959-975. https://doi.org/10.1016/j.joi.2017.08.007 
[10] Arvanitis, R., Villavicencio, D., \& Wei, Z. (2014). L'apprentissage technologique dans les pays émergents. Revue D'anthropologie Des Connaissances, 8, 3(3), 495. https://doi.org/10.3917/rac.024.0495

[11] Arya, A., \& Bhaduri, S. (2018). Technological Capabilities in the Indian Ayurveda Medicine Industry: Lessons for 'Industrialisation' of Alternative Medicine in the Post-WTO Regime. African Journal of Science, Technology, Innovation and Development, 10(3), 287-298. https://doi.org/10.1080/20421338.2018.1455314

[12] Barney, J. B. (1991). Firm Resources and Sustained Competitive Advantage. Journal of Management, 17(1), 99-120. https://doi.org/10.1177/014920639101700108

[13] Barro, R. (1991). Economic Growth in a Cross Section of Countries. The Quarterly Journal of Economics, 106(425), 407-443.

[14] Barro, R., \& Lee, J.-W. (1994). Sources of economic growth. Carnegie-Rochester Conference Series on Public Policy, 40, 1-46. https://doi.org/10.2307/2937943

[15] Barro,R.,\& Sala-i-Martin,X.(2004).Economic Growth. Cambridge:The MIT Press.

[16] Bell, M. (2006). Time and Technological Learning in Industrialising Countries: How Long Does it Take? How Fast Is it Moving (if at All)? International Journal of Technology Management, 36(1/2/3), 25-39. https://doi.org/10.1504/ IJTM.2006.009959

[17] Bell, M., \& Pavitt, K. (1992). Accumulating Technological Capability in Developing Countries. The World Bank Economic Review, 6(suppl 1), 257-281. https://doi. org/10.1093/wber/6.suppl_1.257

[18] Beyhan, B., \& Cetindamar, D. (2013). No Escape from The Dominant Theories: The Analysis of Intellectual Pillars of Technology Management in Developing Countries. Technological Forecasting \& Social Change, 78, 103-115. https://doi. org/10.1007/978-1-4471-4887-6_2

[19] Biggs, T., Shah, M., \& Srivastava, P. (1995). Technological Capabilities and Learning in African Enterprises. World Bank Technical Paper. http://documents1. worldbank.org/curated/en/649901468773714947/pdf/multi0page.pdf

[20] Bowonder, B. (1998). Industrialisation and Economic Growth of India: Interactions of Indigenous and Foreign Technology. International Journal of Technology Management, 15(6-7), 622-644. https://oi.org/10.1504/IJTM.1998.002624 
[21] Brown, F., \& Domínguez, L. (2004). Medición de las capacidades tecnológicas en la industria mexicana. Revista CEPAL, (83), 135-151. https://www.cepal.org/es/ publicaciones/10969-medicion-capacidades-tecnologicas-la-industria-mexicana

[22] Carattoli, M. (2013). Capacidades dinámicas: líneas promisorias y desafíos de investigación. Cuadernos de Administración, 26(47), 165-204. http://www.scielo. org.co/pdf/cadm/v26n47/v26n47a08.pdf

[23] Cass, D. (1965). Optimum Growth in an Aggregative Model of Capital Accumulation. The Review of Economic Studies, 32(3), 233-240. https://doi. $\operatorname{org} / 10.2307 / 2295827$

[24] Chang, H. J. (2012). The Manufacturing Sector and the Future of Malaysia's Economic Development. Journal Pengurusan, 35, 3-12. http://journalarticle.ukm.my/5799/

[25] Chen, L. C. (2013). Jumping Over Latecomers' Upgrading Barriers: The Exploration of China's Upgrading Opportunities By Taiwan's Machine Tool Industry. In Proceeding of the 2013 “Suzhou-Silicon Valley-Beijing” International Innovation Conference: Technology Innovation and Diasporas in a Global Era, SIIC 2013, (pp. 320-325) IEEE. https://doi.org/10.1109/SIIC.2013.6624208

[26] Chica, R. (2007a). Elementos de política de desarrollo productivo. Universidad Autónoma de Manizales.

[27] Chica, R. (2007b). Latinoamérica frente a la globalización: una estrategia alternativa de desarrollo. Universidad Autónoma de Manizales.

[28] Coombs, J. E., \& Bierly, P. E. (2006). Measuring Technological Capability and Performance. $\mathrm{R}$ and $\mathrm{D}$ Management, 36(4), 421-438. https://doi. org/10.1111/j.1467-9310.2006.00444.x

[29] Cusumano, M. A., \& Elenkov, D. (1994). Linking International Technology Transfer With Strategy And Management: A Literature Commentary. Research Policy, 23(2), 195-215. https://doi.org/10.1016/0048-7333(94)90053-1

[30] Dahlman, C. J., Ross-Larson, B., \& Westphal, L. E. (1987). Managing Technological Development: Lessons from the Newly Industrializing Countries. World Development, 15(6), 759-775. https://doi.org/10.1016/0305-750X(87)90058-1

[31] De Pablos, P. O., \& Edvinsson, L. (Eds.). (2020). Intellectual Capital in the Digital Economy. Routledge. 
[32] Dutrénit, G. (2004). Building Technological Capabilities in Latecomer Firms: A Review Essay. Science, Technology and Society, 9(2), 209-241. https://doi. org $/ 10.1177 / 097172180400900202$

[33] Dutrénit, G. (2007). The Transition from Building-Up Innovative Technological Capabilities to Leadership by Latecomer Firms. Asian Journal of Technology Innovation, 15(2), 125-149. https://doi.org/10.1080/19761597.2007.9668640

[34] Dutrénit, G., \& Capdevielle, M. (1993). El perfil tecnológico de la industria mexicana y su dinámica innovadora en la década de los ochenta. El Trimestre Económico, (239), 643-674.

[35] Dutrénit, G., Natera, J. M., Puchet Anyul, M., \& Vera-Cruz, A. O. (2019). Development Profiles And Accumulation of Technological Capabilities in Latin America. Technological Forecasting and Social Change, 145, 396-412. https://doi. org/10.1016/j.techfore.2018.03.026

[36] Enos, J. (1991). The Creation of Technological Capability in Developing Countries, a study prepared for the International Labour Office (ILO) within the framework of the World Employment Programme. Pinter Publishers. https://doi. org/10.1080/08109029408629390

[37] Fessehaie, J. (2013). The Impact of Privatisation on Technological Upgrading: A Case Study. African Journal of Science, Technology, Innovation and Development, 5(6), 499-509. https://doi.org/10.1080/20421338.2013.820456

[38] Fransman, M., \& King, K. (1984). Technological Capability in the Third World. Macmillan. https://link.springer.com/chapter/10.1007/978-1-349-17487-4_1

[39] Figueiredo,P.N.(2014).Beyond TechnologicalCatch-Up: AnEmpirical Investigation of Further Innovative Capability Accumulation Outcomes in Latecomer Firms with Evidence from Brazil. Journal of Engineering and Technology Management, 31, 73-102. https://doi.org/10.1016/j.jengtec man.2013.10.008

[40] Freeman, C. (1994). The Economics of Technical Change. Cambridge Journal of Economics. Oxford University Press. https://doi.org/10.2307/24231814

[41] Gammeltoft, P. (2003). Embedded Flexible Collaboration and Development of Local Capabilities: A Case Study of the Indonesian Electronics Industry. International Journal of Technology Management, 26(7), 743. https://doi. org/10.1504/IJTM.2003.003454 
[42] Gammeltoft, P. (2004). Development of Firm-Level Technological Capabilities: The Case of the Indonesian Electronics Industry. Journal of the Asia Pacific Economy, 9(1), 49-69. https://doi.org/10.1080/13547860310001628294

[43] García, F. E., \& Navas, J. E. (2007). Las capacidades tecnológicas y los resultados empresariales. Un estudio empírico en el sector biotecnológico español. Cuadernos de Economía y Dirección de la Empresa, 10(32), 177-210. https://doi.org/10.1016/ S1138-5758(07)70095-6

[44] Gaviria, M., Merigó, J. M., \& Baier-Fuentes, H. (2019). Knowledge Management: A Global Examination Based on Bibliometric Analysis. Technological Forecasting and Social Change, 140, 194-220. https://doi.org/10.1016/j.techfore.2018.07.006

[45] Gonsen, R. (1998). Tecnhological Capabilities in Developing Countries: Industrial Biotechnology in Mexico. Macmillan. https://doi.org/10.1007/978 -1-349-26369-1

[46] Hansen, U. E., \& Ockwell, D. (2014). Learning and Technological Capability Building in Emerging Economies: The Case of the Biomass Power Equipment Industry in Malaysia. Technovation, 34(10), 617-630. https://doi.org/10.1016/j. technovation.2014.07.003

[47] Hansen, E. U., \& Lema, R. (2019). The Co-Evolution of Learning Mechanisms and Technological Capabilities: Lessons from Energy Technologies in Emerging Economies. Technological Forecasting and Social Change, 140, 241-257. https:// doi.org/10.1016/J.TECHFORE.2018.12.007

[48] Hsu, J. Y., Poon, J. P., \& Yeung, H. W. C. (2008). External Leveraging and Technological Upgrading Among East Asian Firms in the US. European Planning Studies, 16(1), 99-118. https://oi.org/10.1080/09654310701747993

[49] Iguchi, C. (2008). Determinants of Backward Linkages: The Case of TNC Subsidiaries in Malaysia. Asian Business and Management, 7(1), 53-73. https:// doi.org/10.1057/palgrave.abm.9200244

[50] Ince, H., Imamoglu, S. Z., \& Turkcan, H. (2016). The Effect of Technological Innovation Capabilities and Absorptive Capacity on Firm Innovativeness: A Conceptual Framework. Procedia Social and Behavioral Sciences, 235, 764-770. https://doi.org/10.1016/j.sbspro.2016.11.078

[51] İpek, İ. (2019). Organizational Learning in Exporting: A Bibliometric Analysis and Critical Review of the Empirical Research. International Business Review, 28(3), 544-559. https://doi.org/10.1016/j.ibusrev.2018.11.010 
[52] Jonker, M., Romijn, H., \& Szirmai, A. (2006). Technological Effort, Technological Capabilities and Economic Performance: A Case Study of the Paper Manufacturing Sector in West Java. Technovation, 26(1), 121-134. https://doi.org/10.1016/j. technovation.2004.10.002

[53] Jun, I. W., \& Rowley, C. (2019). Competitive Advantage and the Transformation of Value Chains Over Time: The Example of A South Korean Diversified Business Group, 1953-2013. Business History, 61(2), 343-370. https://doi.org/10.1080/00076 791.2018.1430141

[54] aatooKaldor, N. (1970). The Case for Regional Policies. Scottish Journal of Political Economy, 17(3), 337-348.

[55] Kaldor, N. (1961). Capital Accumulation and Economic Growth. The Theory of Capital. Macmillan. https://link. springer.com/chapter/10.1007/978 -1-349-08452-4_10

[56] Kaldor, N. (1975, Dec.). Economic Growth and the Verdoorn Law. A Coment on Mr. Rowthorn's Article. The Economic Journal, 15, 891-896. https://doi. $\operatorname{org} / 10.2307 / 2230633$

[57] Kale, D. (2019). From Small Molecule Generics to Biosimilars: Technological Upgrading and Patterns of Distinctive Learning Processes in the Indian Pharmaceutical Industry. Technological Forecasting and Social Change, 145, 370383. https://doi.org/10.1016/j.techfore.2018.09.020

[58] Kiamehr, M. (2017). Paths of Technological Capability Building in Complex Capital Goods: The Case of Hydro Electricity Generation Systems in Iran. Technological Forecasting and Social Change, 122, 215-230. https://doi. org/10.1016/j.techfore.2016.03.005

[59] Kim, L. (1999). Building Technological Capability for Industrialization: Analytical Frameworks and Korea's Experience. Industrial and Corporate Change, 8(1), 111-136. https://doi.org/10.1093/icc/8.1.111

[60] Kim, L., \& Kim, Y. (1985). Innovation in a Newly Industrializing Country: A Multiple Discriminant Analysis. Management Science, 31(3), 312-322.

[61] Kogut, B., \& Zander, U. (1992). Knowledge of the Firm, Combinative Capabilities, and the Replication of Technology. Organization Science, 3(3), 383-397. https://doi.org/10.1287/orsc.3.3.383 
[62] Kook, S. H., Kim, K. H., \& Lee, C. (2017). Dynamic Technological Diversification and its Impact on Firms' Performance An Empirical Analysis of Korean IT Firms. Sustainability (Switzerland), 9(7). https://doi.org/10.3390/su9071239

[63] Koopmans, T. (1965). On the Concept of Optimal Economic Growth. In T. Koopmans, The Econometric Approach to Development Planning. North Holland.

[64] Lall, S. (1991). Explaining Industrial Success in the Developing World. In V. Balasubramanyam, \& S. Lall (Eds.), Current Issues in Development Economics (pp. 118-155). Macmillan. https://doi.org/10.1007/978-1-349-21587-4_7

[65] Lall, S. (1992). Technological Capabilities and Industrialization. World Development, 20(2), 165-186. https://doi.org/10.1016/0305-750X(92)90097-F

[66] Lall, S. (2001). Competitiveness, Technology and Skills. Edward Elgar Publishing.

[67] Lavarello, P., Mancini, M., \& Sarabia, A. (2017). Respuestas nacionales frente a la desindustrialización. Algunas lecciones de la política industrial de Argentina 2003-2015. Cuadernos del Cendes, 34(95), 67-89.

[68] Lee, C. W., Hayter, R., \& Edgington, D. W. (2010). Large and Latecomer Firms: The Taiwan Semiconductor Manufacturing Company and Taiwan's Electronics Industry. Tijdschrift Voor Economische En Sociale Geografie, 101(2), 177-198. https://doi.org/10.1111/j.1467-9663.2009.00539.x

[69] Liu, S. J. (1998). Industrial Development and Structural Adaptation in Taiwan: Some Issues of Learned Entrepreneurship. IEEE Transactions on Engineering Management, 45(4), 338-348. https://oi.org/10.1109/17.728575

[70] López, A. (2010). Innovation and IPR in a Catch-Up-Falling-Behind Process. Intellectual Property Rights, Development, and Catch Up: An International Comparative Study: An International Comparative Study. Oxford Scholarship Online. https://doi.org/10.1093/acprof:oso/9780199574759.003.0008

[71] Lorentzen, J., Muller, L., Manamela, A., \& Gastrow, M. (2011). Smart Specialization and Global Competitiveness: Multinational Enterprises and Location-Specific Assets in Cape Town. African Journal of Business Management, 5(12), 4782-4791. https://doi.org/10.5897/AJBM10.980

[72] Lucas, R. (1988). On the Mechanism of Economic Development. Journal of Monetary Economics, 22, 3-42.

[73] Lucas, R. (1990). Why Doesn't Capital Flow from Rich to Poor Nations? American Economic Review, 80(2), 92-96. https://www.jstor.org/stable/2006549 
[74] Madanmohan, T. R., Kumar, U., \& Kumar, V. (2004). Import-Led Technological Capability: A Comparative Analysis of Indian and Indonesian Manufacturing Firms. Technovation, 24(12), 979-993. https://doi.org/10.1016/S0166-4972(03)00030-0

[75] Martín, G., Díez-Vial, I., \& Delgado-Verde, M. (2019). Intellectual Capital and the Firm: Evolution and Research Trends. Journal of Intellectual Capital. https://doi. org/10.1108/JIC-12-2018-0221

[76] Martins, V. W. B., Rampasso, I. S., Anholon, R., Quelhas, O. L. G., \& Leal Filho, W. (2019). Knowledge Management in the Context of Sustainability: Literature Review and Opportunities for Future Research. Journal of Cleaner Production, 229, 489-500. https://doi.org/j.jclepro.2019.04.354

[77] Miller, D. (2019). The Resource-Based View of the Firm. In Oxford Research Encyclopedia of Business and Management. https://doi.org/10.1093/ acrefore/9780190224851.013.4

[78] More, R. Z., \& Jain, K. (2013). Innovation and Competitiveness Among the Firms in the Indian Automobile Cluster. Innovation and Development, 3(2), 187-204.

[79] Moreira, A. C. (2008). Upstream Linkages Between Tncs and Local Suppliers: Evidences from Portugal. World Review of Science, Technology and Sustainable Development, 5(1), 28-48. https://doi.org/10.1504/WRSTSD.2008.017811

[80] Myint, M. M., Rasiah, R., \& Singaravelloo, K. (2015). Globalization of Industrialization and its Impact on Clothing Workers in Myanmar. Journal of the Asia Pacific Economy, 20(1), 100-110. https://doi.org/10.1080/13547860.2014.974 330

[81] Oliva, F.L., Couto, M.H.G., Santos, R.F. \& Bresciani, S. (2019). The Integration Between Knowledge Management and Dynamic Capabilities in Agile Organizations. Management Decision, 57(8), 1960-1979. https://doi.org/10.1108/ MD-06-2018-0670

[82] Oyelaran-Oyeyinka, B. (2003). Knowledge Networks and Technological Capabilities in the African Manufacturing Cluster. Science, Technology and Society, 8(1), 1-23. https://doi.org/10.1177/097172180300800101

[83] Pack, H., \& Westphal, L. E. (1986). Industrial Strategy and Technological Change: Theory Versus Reality. Journal of Development Economics, 22(1), 87-128. https://doi.org/10.1016/0304-3878(86)90053-2 
[84] Peerally, J. A., \& Cantwell, J. A. (2012). Changes in Trade Policies and the Heterogeneity of Domestic and Multinational Firms' Strategic Response: The Effects on Firm-Level Capabilities. World Development, 40(3), 469-485. https://doi.org/10.1016/j.worlddev.2011.09.020

[85] Pérez-Escatel, A., \& Pérez, V. (2009). Competitividad y acumulación de capacidades tecnológicas en la industria manufacturera mexicana. Investigación Económica, 68(268), 159-187.

[86] Pérez-Hernández, C. C. (2020). Drivers of Technological Capability in Mexico: The Mesoeconomic Factors that Impulse the Techno-Scientific Products. Contaduria $y$ Administración, 65(1). https://doi.org/10.22201/FCA.24488410E.2019.2057

[87] Poon, J. P. H., \& Sajarattanochote, S. (2010). Asian Transnational Enterprises and Technology Transfer in Thailand. European Planning Studies, 18(5), 691-707. https://doi.org/10.1080/09654311003594026

[88] Popli, M., Akbar, M., Kumar, V., \& Gaur, A. (2017). Performance Impact of Temporal Strategic Fit: Entrainment of Internationalization with Pro-Market Reforms. Global Strategy Journal, 7(4), 354-374. https://oi.org/10.1002/gsj.1160

[89] Porter, M. (1990). La ventaja competitiva de las naciones. Vergara.

[90] Ramsey, F. (1928). A Mathematical Theory of Saving. The Economic Journal, 38(152), 543-559. https://www.jstor.org/stable/2224098

[91] Rasiah, R. (2004). Foreign Firms, Technological Capabilities and Economic Performance.Edward ElgarPublishing. https://doi.org/10.1016/j.asieco.2005.08.008

[92] Rebelo, S. (1991). Long-Run Policy Analysis and Long-Run Growth. The Journal of Political Economy, 99(3), 500-521. https://www.jstor.org/stable/2937740

[93] Reddy, N. M., \& Zhao, L. (1990). International Technology Transfer: A Review. Research Policy, 19(4), 285-307. https://doi.org/10.1016/0048-7333(90)90015-X

[94] Santos, J., Zawislak, P., Franzoni, G., \& Vieira, H. (2015). Searching for a Path: A Bibliometric Study on Innovation and Technological Capabilities. International Journal of Innovation 58. https://doi.org/10.5585/iji.v3i2.58

[95] Shi, Y. (2005). Technological Capabilities and Foreign Direct Investment in China. Resources, Technology and Strategy, 10, 243. https://doi. org/10.4324/9780203982259 
[96] Solow, R. (1956). A Contribution to the Theory of Economic Growth. Quarterly Journal of Economics, 70(1), 65-94. https://doi.org/10.2307/1884513

[97] Sussangkarn, C., \& Manupipatpong, W. (2015). A View from ASEAN. In M. Kawai, Y. Park, \& C. Wyplosz (Eds.), Monetary and Financial Cooperation in East Asia. Oxford University Press. https://doi.org/10.1093/ acprof:oso/9780198714156.001.0001

[98] Swan, T. (1956). Economic Growth and Capital Accumulation. Economic Record, 32(2), 334-361. https://doi.org/10.1111/j.1475-4932.1956.tb00434.x

[99] Teece, D., \& Pisano, G. (2003). The Dynamic Capabilities of Firms. In Handbook on Knowledge Management (pp. 195-213). Springer. https://link.springer.com/ chapter/10.1007/978-3-540-24748-7_10

[100] United Nations Industrial Development Organization. (2017). Industrial Development Report 2018: Demand for Manufacturing: Driving Inclusive and Sustainable Industrial Development. UNIDO. https://www.unido.org/sites/default/ files/files/2017-11/IDR2018_OVERVIEW_ENGLISH.pdf

[101] Usawa, H. (1965). Optimum Technical Change in An Aggregative Model of Economic Growth. International Economic Review, 6(1), 18-31. https://www.jstor. org/stable/2525621

[102] Villavicencio, D., \& Arvanitis, R. (1994). Transferencia de tecnología y aprendizaje tecnológico. Reflexiones basadas en trabajos empíricos. El Trimestre Económico, 61(2)(242), 257-279.

[103] Wong, P. K., \& Mathews, J. (2005). Competitive Strategies of Asian High-Tech Firms: The Challenge of Late-Industrialisation. International Journal of Technology Management, 29(1-2), 1-5. http://scholarbank.nus.edu.sg/handle/10635/44381

[104]Zhou, H. J., Wu, D., Wu, X. B., \& Peng, X. M. (2009). Strategic Technology Alliance and Rapid Accumulation of Technological Capabilities in Emerging Economies. IEEM 2009-IEEE International Conference on Industrial Engineering and Engineering Management, 39-43. https://doi.org/10.1109/IEEM.2009.5373461

[105]Zuo, L., Fisher, G. J., \& Yang, Z. (2019). Organizational Learning and Technological Innovation: The Distinct Dimensions of Novelty and Meaningfulness that Impact Firm Performance. Journal of the Academy of Marketing Science, 47(6), 11661183. https://doi.org/10.1007/s11747-019-00633-1 